\title{
Assessing the Force and Timing control of Children with Motor Problems using Elastic Displays
}

\author{
Franceli L. Cibrian \\ Computer Science Department \\ CICESE, Ensenada, Mexico \\ franceli@cicese.edu.mx
}

\author{
Jesus A. Beltran \\ Computer Science Department \\ CICESE, Ensenada, Mexico \\ abeltran@cicese.edu.mx
}

\author{
Monica Tentori \\ Computer Science Department \\ CICESE, Ensenada, Mexico \\ mtentori@ cicese.mx
}

\begin{abstract}
Children with motor problems have difficulties to control their timing and strength. For the last four years, we have been developing BendableSound, an elastic display that enables users to play sounds when interacting with a fabric. In this paper, we report a set of measurements and coordination tasks that we integrated into BendableSound to be used to measure strength and timing control. During a deployment study with 22 children with autism presenting motor problems, we found out that the scores of the coordination tasks in BendableSound are correlated with the scores of a clinical tool measuring coordination. This result could mean that elastic displays could be used as an assessment tool for children with motor problems.
\end{abstract}

\section{Author Keywords}

Elastic display; autism; motor control; assessment tool

\section{ACM Classification Keywords}

H.5.m. Information interfaces and presentation (e.g., HCI): Miscellaneous

\section{INTRODUCTION}

Children with motor problems may have limited motor control defaulting their ability to appropriately redirect their movements to respond to sensory stimuli or control their strength [8]. For the last 4 years, we have been developing BendableSound [2] (Figure 1), an elastic display encouraging children with motor problems to improve their timing and force regulation while practicing motor movements when touching a fabric to play sounds.

In this paper, we describe the development and integration into BendableSound of the timing synchronization and control tasks that are used to measure strength and timing control. During these tasks, we estimate features of qualities of the push movement related to timing and force regulation when users push the fabric. We hypothesize that these
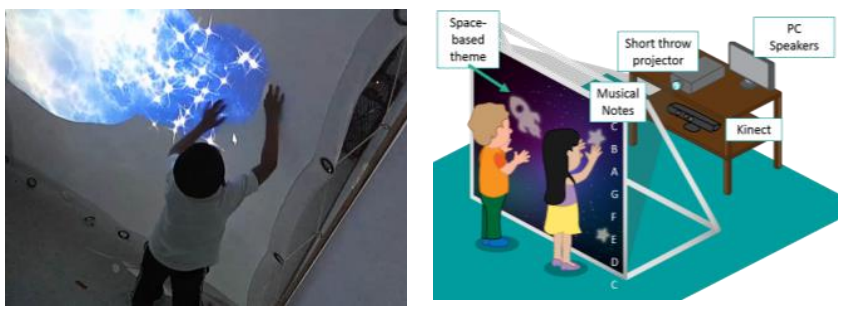

Figure 1. The BendableSound prototype. A child discovering sounds and the space nebula (left). A mock-up of BendableSound (right).

features have a relationship with the control of movements of children with motor problems. We selected the Developmental Coordination Disorder Questionnaire $(\mathrm{DCDQ})^{1} \quad$ [10] as the clinical screening tool measuring coordination and motor problems.

We evaluated our proposal by comparing the results of the DCDQ against a set of indirect features calculated with BendableSound of 22 children with severe autism with motor problems. We chose to evaluate our prototype with children with autism as the prevalence of motor impairments is higher in this population [8].

The main contributions of this work are:

- A set of 3D movement features that could be calculated from interactions with an elastic display.

- Evidence showing BendableSound could be used as an assessment tool to measure timing and force regulation in children with autism.

\section{RELATED WORK}

In this section, we describe research related to the design of elastic displays, and research related to the evaluation of motor movements and gesture analysis.

\section{Design of Elastic displays}

Elastics displays have been proposed to support navigation [11], and manipulate physical [4] and multimedia [1] information. Users can either use one finger, their complete hand, or unimanual and bimanual gestures [4, 9]. For example, DepthTouch [4] is an elastic display mimicking the

\footnotetext{
${ }^{1}$ DCDQ is considered a valid clinical screening tool for children who have coordination challenges (overall sensitivity $=85 \%$; overall specificity $=$ $71 \%$ ). The DCDQ has 15 questions, six related to measuring control during movement, four about fine motor skills and five related to general coordination. Each question must be scored on a 5 point Likert scale. DCDQ.
} 
gravitational forces between spheres. The spheres react according to the force applied when users push the elastic surface. The use of DepthTouch shows the elastic display is easy to understand and help users to quickly create a mental model of how its interaction works [4]. These projects suggest that the affordances of elastic displays help users to understand how to interact with the surface quickly.

\section{Analysis of motor movements and gestures}

Other researchers have explored how to uncover potential interactions with elastic displays [9]. For example, a laboratory study, where 17 young adults used a small-scale elastic display to perform predefined touch-based interactions, found that the affordances of elastic displays enable users to interact with the canvas by pulling it, pushing it or twisting it [9]. These interactions could offer 3D information about the movements of users.

Moreover, research has been focused on the automatic recognition and evaluation of emotion and affective states [5, 7] using the $3 \mathrm{D}$ information of users' movements. For example, in [5], authors introduced a set of 3D movement features that could be computed from the Kinect's skeleton. Such features could potentially help to identify emotions of children with autism by calculating the kinetic energy, symmetry, directness, and fluently of their movements. A preliminary study, comparing the calculated 3D movement features with the video of seven young adults mimicking six basic emotions found those features could give a better description of emotions.

This research may show that elastic displays could provide information that could help us gain an understanding of their users' motor behavior. However, little has been said if such information could be calculated with a challenging population like children with motor problems such as children with severe autism.

\section{THE BENDABLESOUND PROTOTYPE}

BendableSound is a large-scale elastic display that allows users to make music when tapping and touching on top of the fabric canvas (Figure 1-up). Users can play the sounds of different musical instruments. BendableSound displays a 3D background showing an animation of a nebula with translucent space-based elements, like stars and planets, appearing on top of the fabric [2].

The following activities were integrated into BendableSound to use it as an assessment tool. These tasks are guided by a clinical motor tests reported in [3].

\section{Timing synchronization task}

For the timing synchronization task, BendableSound generates pacing tones (50 ms duration, $1 \mathrm{~s}$ interval) acting as a music pulse. Children need to push and synchronize their movements with the pacing tones. To complete a trial,
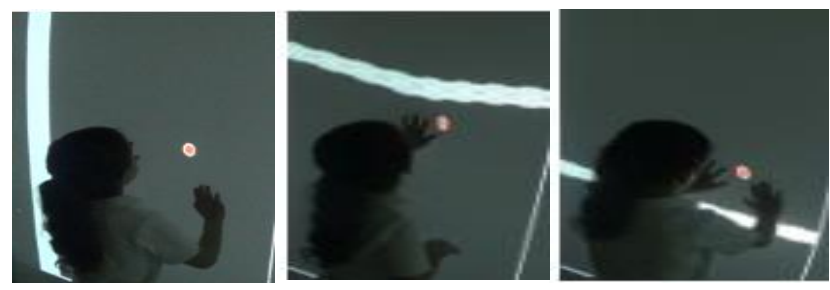

Figure 2. A girl using BendableSound during the timing task (left), and the Strength control task (center-right)

children must complete five repetitions listening to the tones and another five repetitions without the tones.

Children must complete five trials (Figure 2-left).

\section{Strength control task}

For the strength control task, children need to match the force exerted by the push movement with a target. BendableSound presents a target in the form of a horizontal line and a rocket that moves vertically and proportionally to the amount of force children use when pushing the fabric. When children push the fabric, the rocket flies to reach the horizontal line. The horizontal line first appears at three-quarters of the height of the display (Figure 2-center) demanding from children to push harder. Then, it appears a quarter of the height to ask children to push in a more soft way (Figure 2right). To complete a trial, children need to complete five repetitions with and without the visual feedback, and five trials pushing hard and then pushing soft.

For both the timing and strength tasks, the therapist may interrupt the trials as needed.

\section{Developing BendableSound}

BendableSound uses a $1.5 \times 2$ m elastic display and a DLP ultra-short-throw projector displaying a $1.4 \times 1.4 \mathrm{~m}$ image. The Kinect sensor is placed $1.78 \mathrm{~m}$ behind the fabric, and $1 \mathrm{~m}$ over the floor (Figure 1-right). BendableSound uses the TSPS $^{2}$ v 1.3.7 library to get the data captured by the Kinect sensor [2]. We used the Processing language to develop the visual and auditory feedback and to store the logs of usage along with all the data captured by the Kinect -each frame stored of data encompasses 60 fps. We logged all user interactions with the fabric. Each record on the $\log$ is the location of the push movement in a time $t$ (Figure 3):

$$
\overrightarrow{r_{t}}=x_{t} \hat{\imath}+y_{t} \hat{\jmath}+z_{t} \hat{k}
$$

where, $x_{t}, y_{t}, z_{t}$ are the size of the location of one push movement in a time $t$, in each direction; whereas $\hat{\imath}, \hat{\jmath}, \hat{k}$ are the unitary vectors in the direction of each axis.

\section{FORCE AND TIMING FEATURES}

To analyze the logs, Table 1 shows a summary of the features calculated from the 3D push movements tracking information captured by the Kinect. An example of input data can be seen in Figure 3. These measures were selected as children with motor problems present more variability in

${ }^{2}$ http://www.tsps.cc/ 


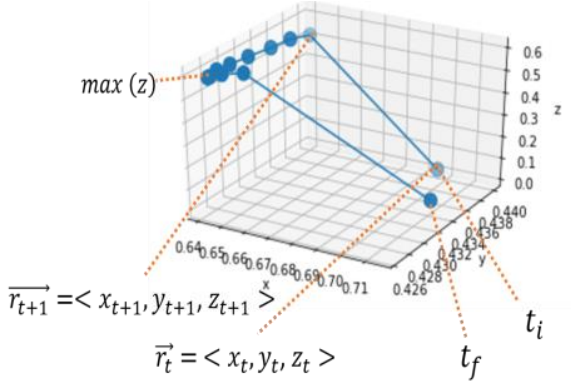

Figure 3. An example of input data of one push movement their inter-response interval, force, and they react early or late when responding a stimuli [6].

\section{EVALUATION}

To evaluate if the features computed by BendableSound are as efficient as the DCDQ, we conducted a deployment study at Pasitos, a school-clinic attending children with autism.

\section{Methods}

We recruited 22 children with severe autism with motor problems (aged between 4 to 8 years old). We also hired one psychologist trained in using the DCDQ. We equipped two rooms at Pasitos: one with BendableSound, and the other one with "sports" material, like balls and toys. The procedure was as follows:

- Assessments. For up to 15 minutes, participants first used BendableSound and completed the timing synchronization and strength control tasks. Then, participants conducted "sports-alike" activities, like throwing a ball; so, the psychologist could answer the DCDQ.

- Therapy. Next, participants completed eight sessions of music therapy.

- Assessments. Finally, participants conducted the assessment activities again using BendableSound and using the "sports" material to answer the DCDQ.

In total, we collected 44 logs of participants using the timing and force task, and the scores to the DCDQ for each participant. To analyze this data we calculated, for each participant:

- The mean of all the features (Table 1) for every pushing movement each child did per trial.

- The average of the inter-response intervals (timing synchronization).

- The average difference between the applied forces by the children when pushing hard vs. soft (strength).

Then, we calculated the Spearman's rank correlation of the task scores and "control during movement" score of the DCDQ -we took into consideration the section of the DCDQ related to "control during movement" as it was the relevant one for this analysis.

\section{Results}

Overall, our results show that there is a weak correlation between our features and the section we used from the DCDQ.

\begin{tabular}{|c|c|c|c|}
\hline Name & Description & Formula & Variables \\
\hline $\begin{array}{l}\text { Inter- } \\
\text { response } \\
\text { intervals }(I R)\end{array}$ & $\begin{array}{l}\text { The IR is the difference of time between two consecutive push } \\
\text { movements produced without auditory feedback. The IR is better } \\
\text { when the measure is closer to the interval of the pacing tones. }\end{array}$ & $I R=t_{i}-t_{i-1}$ & $\begin{array}{l}t_{i} \text { is the initial time of the } \\
\text { current push movement } \\
t_{i-1} \text { is the initial time of the } \\
\text { previous push movement. }\end{array}$ \\
\hline $\begin{array}{l}\text { Reaction time } \\
(R T)\end{array}$ & $\begin{array}{l}\text { The RT is how long it took the user to touch the fabric after hearing } \\
\text { an auditory tone. }\end{array}$ & $R T=t_{i}-t_{\text {ant } B S}$ & $\begin{array}{l}t_{\text {antBS }} \text { is the time of the } \\
\text { auditory feedback after the } t_{i}\end{array}$ \\
\hline Distance $(d)$ & $\begin{array}{l}\text { The distance is the estimation of the trajectory length drawn in the } \\
\text { space of one push movement. Given that the TSPS library get the } \\
\text { measurements in a range of }[0,1] \text {, to compute the distance per axis, } \\
\text { the values should be changed to the basic magnitude of movement, } \\
\text { in our case meters (reference system). Then, The displacement of } \\
\text { the touch movement in a time } t \text { from a time } t+1 \text { fill be } \\
\overrightarrow{r_{t+1}}=<x_{t+1}, y_{t+1}, z_{t+1}>\text { (See Figure } 3 \text { ). }\end{array}$ & 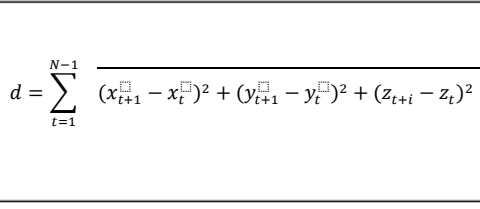 & $\begin{array}{l}x_{t}, y_{t}, z_{t} \text { the size of the } \\
\text { location of one push } \\
\text { movement in a time } t\end{array}$ \\
\hline Velocity $(|\vec{v}|)$ & $\begin{array}{l}\text { To estimate the speed, we calculated the distance between the time } \\
\text { that a user took to conduct the movement. }\end{array}$ & $|\vec{v}|=\frac{d}{t_{f}-t_{i}}$ & $\begin{array}{l}t_{f} \text { is the final time of the } \\
\text { current push movement }\end{array}$ \\
\hline $\begin{array}{l}\text { Acceleration } \\
(|\vec{a}|)\end{array}$ & $\begin{array}{l}\text { The average acceleration is computed by how much the velocity of } \\
\text { the push movement changed during the time taken in the push } \\
\text { movement }\end{array}$ & $|\vec{a}|=\frac{|\vec{v}|}{t_{f}-t_{i}}$ & \\
\hline Mass & $\begin{array}{l}\text { To estimate the mass needed to push the elastic fabric: First, we } \\
\text { used a dynamometer to pull the elastic fabric at different points } \\
\text { (i.e., near to the edges of the fabric, and at the center). Second, we } \\
\text { estimated the relationship between the amounts of mass given by } \\
\text { the dynamometer and the depth of the push movement. Then, we } \\
\text { infer how much mass is needed to get the max depth }(\max (z))\end{array}$ & $\operatorname{mass}=\max (z) * k g$ & $\begin{array}{l}\max (z) \text { is the max depth } \\
\text { getting with a push } \\
\text { movement } \\
\mathrm{kg} \text { represents the proportion } \\
\text { of kilograms needed to push } \\
\text { the fabric until the } \max (\mathrm{z}) \text {. }\end{array}$ \\
\hline Force $(f)$ & $\begin{array}{l}\text { To have an estimation of the force used to push the elastic display, } \\
\text { we calculate the acceleration } a \text { of the push movement by multiplied } \\
\text { the mass needed to get to the max depth. Also, we compute the } \\
\text { time needed to arrive the max depth }(t f) \text {, and how much time the } \\
\text { child spent on the max depth }(d f)\end{array}$ & $\begin{array}{c}f=\operatorname{mass} *|\vec{a}| \\
t f=t_{i \max (z)}-t_{i} \\
d f=t_{f \max (z)}-t_{\max (z)}\end{array}$ & $\begin{array}{l}t_{\text {ifmax }}, \text { is the initial time } \\
\text { where the chid arrives to the } \\
\max (z) \\
t_{f \max (z)} \text { is when the user } \\
\text { left these position. }\end{array}$ \\
\hline
\end{tabular}

Table 1. Force and timing features calculated of the interactions with an elastic display 
For the timing synchronization task, we found out that there is a negative correlation between the mean of inter-response intervals and the control of movement scores $(\mathrm{rho}=-0.428$; $\mathrm{p}=0.003$; Figure 4-down). This result could mean that participants who touched the fabric more rhythmically have better synchronization of their movements -as their DCDQ score is higher.

For the strength control task, we found out that there is a weak positive correlation between the average difference of the force applied when pushing soft and hard with the score of the control of their movements (rho $=0.316 ; p=0.036$; Figure 4-up). This could mean that, if the difference between pushing soft and hard is bigger, then, participants understood concepts related to self-regulation of force -as their DCDQ score is also higher.

\section{CONCLUSIONS}

Overall, these results could show that, although many variables could affect the control of movements of children with autism, timing synchronization, and the strength control are two of them.

We found out that the scores of the tasks evaluated with the elastic display have a weak but statistically significant correlation with the clinical screening DCDQ. This could mean that the features computed with an elastic display could be used to understand and assess the timing synchronization and the strength control of children with severe autism.

Elastic displays might help parents and therapist to assess the progress in both, timing and strength regulation of children's movements, in a more engaging and fun way. Elastic displays may also be helpful in monitoring the therapy, modifying it according, or reporting results in an easy way.

As future work, we are planning to investigate the efficacy of using BendableSound as a therapy for children with autism. Also, we would like to explore more features that could be calculated from user interactions with an elastic display and further understand if children with motor problems have a "distinctive" gestural pattern when using elastic displays.

\section{REFERENCES}

[1] Cassinelli, A. and Ishikawa, M. 2005. Khronos projector. ACM SIGGRAPH 2005. 10.

[2] Cibrian, F. L., Pena, O., Ortega, D. \& Tentori, M. 2017. BendableSound: Designing a Fabric-based Interactive Surface to Support Neurologic Music Therapy sessions for children with severe autism. Int. J. Hum. Comput. Stud. 107, (2017), 22-37.

[3] Lundy-Ekman, L. et al. 1991. Timing and force control deficits in clumsy children. J. of Cog. Neuroscience. 3, 4 (1991), 367-376.

[4] Müller, M. et al. 2015. Data exploration on elastic displays using physical metaphors. $x \operatorname{CoAx} 2015$, 111-124.

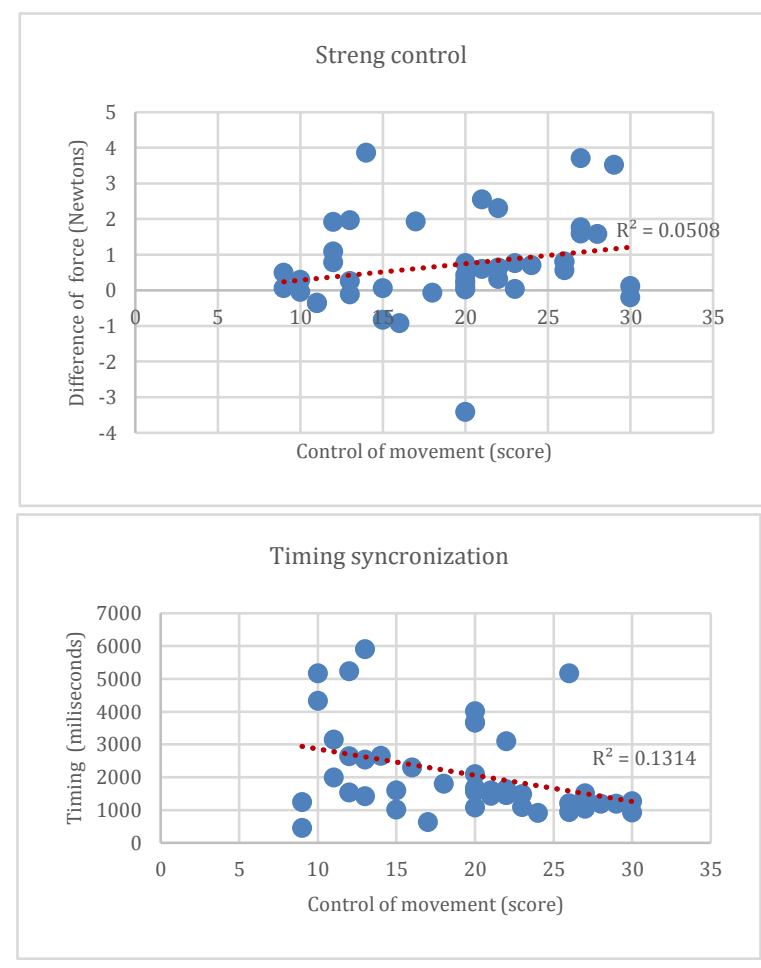

Figure 4. The Control of movement scores against the difference of force (up) and timing score (down)

[5] Piana, S. et al. 2013. A set of Full-Body Movement Features for Emotion Recognition to Help Children affected by Autism Spectrum Condition. IDGEI. (2013), 1-7.

[6] Piek, J.P. and Skinner, R.A. 1999. Timing and force control during a sequential tapping task in children with and without motor coordination problems. J. of the Int. Neuropsychological Society. 5, 4 (1999), 320-329.

[7] Rivas, J.J. et al. 2015. Detecting affective states in virtual rehabilitation. PervasiveHealth'15, 287-292.

[8] Staples, K.L. and Reid, G. 2010. Fundamental movement skills and autism spectrum disorders. $J$. aut. and develop. disor. 40, 2, 209-17.

[9] Troiano, G.M. et al. 2014. User-defined gestures for elastic, deformable displays. AVI '14 (2014), 1-8.

[10] Wilson, C. 2009. THE DEVELOPMENTAL COORDINATION DISORDER QUESTIONNAIRE 2007 (DCDQ'07). Physical \& Occupational Therapy in Pediatrics. 29, 2 (2009), 182-202.

[11] Yun, K. et al. 2013. ElaScreenn: Exploring multidimensional data using elastic screen. CHI EA '13. (2013), 1311. 